proposal to initiate an improvement project. Structured education and training was carried out by the champions in the multidisciplinary group. Every month, a consultant neonatologist and clinical scientist in microbiology reviewed the infections for diagnosing CLABSI. Data is displayed in the unit to feedback to the staff. The initial timescale for change was 4 months.

Measurement of improvement The measurement for improvement was done through monitoring process and outcome measures. The process measures monitored were compliance with 1) hand hygiene 2) using insertion and maintenance checklists 3) changing of dressings on surgically inserted CL once a week 3) changing of hubs twice a week and 4) removing lines when not required. The data was analysed using Statistical Process Charts. Run charts were used for process measures and control charts were used for outcome measures.

Between December 2010 and June 2014, 350 children had 571 central lines during their stay. Of these, 315 were peripherally inserted central catheters (PICC), 161 were surgically inserted central catheters (SICC) and 95 umbilical lines. Together they amounted to 12,204 CLD. There were 53 CLABSI resulting in the overall rate of $4.3 / 1000$ CLD. However, the base line rate between December 2010 and March 2011 was 8.3/1000 CLD and post intervention the rate has fallen to $3.7 / 1000$ CLD (Figure 1).

Effects of changes The CLABSI rate was reduced by $55 \%$. It could be inferred that in a similar proportion of patients it may have reduced the morbidity and length of stay. It is suggested that each CLABSI increases the length of stay by 3 weeks and cost of care by $£ 10000$.

Due to problems with person dependency, CLABSI rate increased in the second year but once this issue was addressed, improvement was regained.

Lessons learnt We learnt to avoid person dependency and build CL surveillance into routine daily work.

Message for others Through this project we demonstrated that improvement methodology has a potential role in ensuring reliable delivery of evidence based medicine and improving outcomes.

\section{G541(P) IMPROVING THE EFFICIENCY OF PROCEDURE LISTS IN PAEDIATRIC HAEMATOLOGY-ONCOLOGY - A QUALITY IMPROVEMENT PROJECT}

F Kagalwala, H Salam, V Grandage, H Burgess, L Brown. Paediatric Oncology, University College London Hospitals NHS Trust, London, UK

\subsection{6/archdischild-2015-308599.492}

Background Childhood cancer incidence is increasing in the United Kingdom with an average of 1574 new cases diagnosed per year. Bone marrow aspiration and delivery of intrathecal chemotherapy are routine procedures in paediatric haematologyoncology. National Institute of Clinical Excellance (NICE) Improving Outcomes in Children and Young People with Cancer acknowledges the importance of dedicated theatre slots to reduce delays in diagnosis and therapy. To ensure high quality safe patient care efficient organisation and smooth running of procedure lists is paramount.

Context This Quality Improvement Project was carried out in the Paediatric haematology-Oncology department of a tertiary oncology hospital over 5 months. It involved oncology doctors, the anaesthetics, oncology day care nurses, theatre staff, porters and laboratory staff.

Problem There were frequent delays in starting the list and interruptions resulting in the list overrunning causing staff and patient dissatisfaction and late arrival of bone marrow and lumbar puncture samples to the laboratory.

Assessment of problem and analysis of its causes Meetings with stakeholders were held to discuss the current status, identify obstacles and possible solutions. An initial audit over one month was performed to identify the start and finish times and document the reasons for delay.

The audit highlighted the following:

1. Inadequate training and use of staff resources.

2. Poor time management

3. Inadequate booking system for appointments

4. Late sample delivery to the laboratory

Abstract G540(P) Figure 1 Rate of Central line associated blood stream infection per 1000 central line days 
Study design This was a qualitative observational study with analysis of pre and post interventional audit results.

Intervention and Strategy for Change Multidisciplinary communication with various stakeholders was key in agreeing common goals to improve efficiency and quality of the current theatre list. Using a Plan-do-Study-Act Cycle changes were gradually introduced over the next 4 months. The teams were updated by regular weekly emails and monthly meetings to discuss the next steps.

Staff Resources: Rota allocation to ensure optimal junior medical staff cover on the procedure days to allow prompt start. Improved training of doctors and allocating two doctors to a procedure list, one of whom is an experienced senior doctor who can provide immediate assistance to the junior colleague. In addition an Advanced Nurse Practioner has been appointed to start a nurse led service. Improved communication with anaesthetic collegues and availability of their contact details.

Time management: Inter-patient delays were approached by improved co-ordination amongst the recovery and day care nurses and patient transport strategies.

Booking system: With increasing patient numbers it was essential to have an improved electronic booking system. An appointment scheduler giving staggered appointment times would be introduced. Pending this, patients were encouraged to arrive before 10 am and have necessary investigations done in time prior to their procedure.

Sample delivery: Introduction of specific porters for timely collection of samples enabling quick diagnosis of new oncology children. Re-organisation of the procedure order so that diagnostic bone marrows are first on the list.

Measurement and effects of change Descriptive statistics was used and nominal variables calculated as percentages. The results are:-

1. $85 \%$ reduction in late finish and cancelled procedures

2. $100 \%$ of the samples delivered to laboratory by $5 \mathrm{pm}$

3. $80 \%$ improvement in availability of pre-procedure blood results

4. Improved training of doctors and team morale

Lessons learnt Inclusion of patient satisfaction surveys would have enabled us to include their views and make modifications to the service.

Message for others Good planning with adherence to SMART objectives is essential

Early involvement of stake holders and agreement on common goals

To ensure sustainability we involved the permanent day care nurses and senior managers.

\section{G542(P) PLANNING AHEAD: IMPLEMENTATION OF RESPIRATORY SUPPORT FOR BRONCHIOLITIS AT DESIGNATED WARD AREAS OUTSIDE PICU BEFORE THE SEASON}

B Tharayil, D Jyothish. General Paediatrics, Birmingham Children's Hospital, Birmingham, UK

\subsection{6/archdischild-2015-308599.493}

Context The General Paediatric department in the major children's teaching hospital is a large and busy unit with Paediatric intensive care unit (PICU) on-site. There are approximately 4300 admissions per annum. In-patient numbers vary considerably between the seasons with an average range of $80 \pm 10$ in the winter.

Problem A significant number of winter admissions where infants with bronchiolitis and a proportion require respiratory support with high flow nasal cannula oxygen therapy (HFNC).

Assessment of problem and analysis of its causes With this in mind we analysed the existing bronchiolitis patient pathway. We identified HFNC is only provided in PICU due to staff competencies. This has lead to loss of PICU bed resulting in cancellation of elective cardiac surgeries and refusal of retrievals.

Intervention A focus group was formed following the analysis to look at the feasibility of HFNC outside PICU. A strategy with a combined focus on safety and effective treatment with HFNC for children with bronchiolitis, nursed in designated Medical High Dependency Unit (MHDU) beds was developed.

Study design The 'Plan Do Study Act'(PDSA) cycle guided iterative stages of the HFNC project development. The normalisation process theory helped to introduce the new intervention providing the right treatment for the right patient at the right time without delay.

Strategy for change The equipment designed specifically for delivering humidified nasal high flow with in-built safety alarm was identified and it was procured for the HFNC project. The designated MHDU beds were identified on the same floor at one place rather than spreading it out across. We identified the optimum and relevant staffs; nurses, doctors and advance nurse practitioners (ANP); to be trained after estimating the capacity and demand for services to manage the new change safely. More than $90 \%$ of medical team was trained and all nurses in designated areas were also trained over the period with multiple dedicated sessions by three trainers supported by the representative from the company. A hospital grand round presentation was done before starting the project. An intranet hospital link was developed with training materials and standard operating procedure (SOP). A DVD guide showing all aspects of HFNC was also developed and included in the link.

Measurement of improvement We are currently in the stage of seeing the positive effects of HFNC project and data is being collected prospectively.

Effects of changes A collaborative approach was undertaken for the improvement of HFNC for bronchiolitis outside PICU. The process involved clinicians, nurses and managers in the redesign of bronchiolitis treatment pathway. By maintaining and adhering to a standardised operating document, a culture of continuous re-inforcement and support with clear guideline and consistency helped in the implementation of this new change and effective treatment successfully in the bronchiolitis patient pathway.

Lessons learnt There were barriers like competing priority of the professionals involved to achieve the same goal, clarity of role in delivering change and accountability and training of relatively large number the staff.

Message for others It is important to ensure stakeholder engagement from the outset at the start. Having trust, rapport and confidence with the wider team have hugely helped for the acceptance without any hindrance. It is very crucial to recognise the potential pitfalls and develop preventative actions accordingly. The task for this project was not to fixate on the cost side of the equation - saving money or making cuts - but to improve the productivity. The focus was on the benefits side of providing the right treatment for the right patient at designated place safely thereby increasing the value of care to patients. 